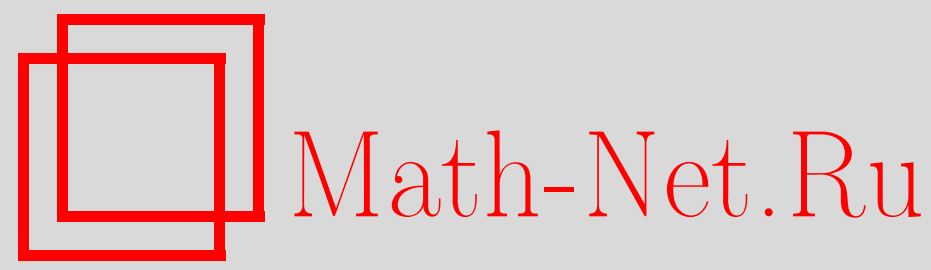

Г. М. Бродский, О модулях, решеточно изоморфных линейно компактным, Матем. заметкu, 1996, том 59, выпуск 2, 174-181

DOI: https://doi.org/10.4213/mzm1704

Использование Общероссийского математического портала MathNet.Ru подразумевает, что вы прочитали и согласны с пользовательским соглашением

http://www . mathnet.ru/rus/agreement

Параметры загрузки:

IP : 44.207 .124 .84

26 апреля 2023 г., $17: 34: 39$

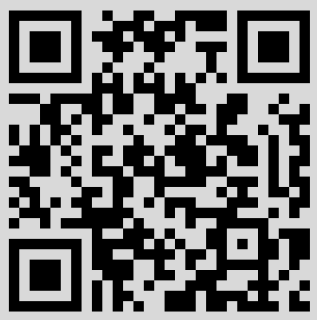


ТОм 59 выПУСК 2 фЕВРАль 1996

\section{О МОДУЛЯХ, РЕШЕТОЧНО ИЗОМОРФНЫХ ЛИНЕЙНО КОМПАКТНЫМ}

\section{Г.М. Бродский}

Рассматриваются ассоциативные кольца с единицей и унитарные модули, гомоморфизмы записьваются с противоположной стороны от скаляров.

Для модуля $X$ через $L(X), \operatorname{End}(X), E(X), \operatorname{Soc}(X)$ и $J(X)$ условимся обозначать его решетку подмодулей, кольцо эндоморфизмов, инъективную оболочку, цоколь и радикал Джекобсона. Модули $X$ и $Y$ (вообще говоря, над разными кольцами) называется решеточно изоморфнымми, если изоморфны решетки $L(X)$ и $L(Y)$. Аналогично определяются решеточно антиизоморфные модули. Модуль $X$ называется линейно компактным (в дискретной топологии), если для любого семейства $\left(x_{\lambda}\right)_{\lambda \in \Lambda}$ элементов модуля $X$ и любого семейства $\left(X_{\lambda}\right)_{\lambda \in \Lambda}$ подмодулей в $X$ из разрешимости всякой конечной подсистемы сравнений $x \equiv x_{\lambda}\left(\bmod X_{\lambda}\right), \lambda \in \Lambda$, вытекает существование решения всей системы.

Цель настоящей заметки - охарактеризовать модули, решеточно изоморфные линейно компактным. Попутно обнаруживается невозможность определения линейно компактного модуля в терминах решетки его подмодулей. Отметим в этой связи, что линейная компактность модуля допускает теоретико-категорные описания (см. [1, предложение 1.4$]$; [2, теоремa 29.7]).

Приведем необходимые определения и обозначения. Модуль $X$ назьвается АВ5*-модулем, если $Y+\bigcap_{\lambda \in \Lambda} Y_{\lambda}=\bigcap_{\lambda \in \Lambda}\left(Y+Y_{\lambda}\right)$ для всех подмодулей $Y$ и направленных вниз семейств подмодулей $\left(Y_{\lambda}\right)_{\lambda \in \Lambda}$. Подмодуль $Y$ модуля $X$ назьвается конечным, если для любого семейства $\left(Y_{\lambda}\right)_{\lambda \in \Lambda}$ подмодулей в $X$ условие $Y=\bigcap_{\lambda \in \Lambda} Y_{\lambda}$ влечет $Y=\bigcap_{\lambda \in \Theta} Y_{\lambda}$ для некоторого конечного подмножества $\Theta \subseteq \Lambda$. Модуль $X$ назьвается конечно копорожденным, если его нулевой подмодуль 0 коконечен. Модуль $X$ называется конечномерным в смысле Голди, если любое независимое семейство его ненулевых подмодулей конечно. Модуль $X$ называется 
QFD-модулем, если все его фактормодули конечномерны в смысле Голди. Семейство $\left(Y_{\lambda}\right)_{\lambda \in \Lambda}$ подмодулей модуля $X$ называется конезависимым, если $Y_{\mu}+\bigcap_{\lambda \in \Theta \backslash\{\mu\}} Y_{\lambda}=X$ для любых непустого конечного подмножества $\Theta \subseteq \Lambda$ и элемента $\mu \in \Theta$. Модуль $X$ назьвается коконечномерным (в смысле Такеучи [3]), если любое конезависимое семейство его собственных подмодулей конечно. Модуль $X$ назовем SCD-модулем, если все его подмодули коконечномерны.

Всюду ниже $\left(E_{i}\right)_{i \in I}$ - семейство инъективньх оболочек всех представителей классов изоморфных простых правых $R$-модулей и $U=$ $E\left(\bigoplus_{i \in I} E_{i}\right)$ - наименьший инъективньй кообразующий категории правых $R$-модулей. Пусть $M_{R}$ - произвольньй модуль. Положив $S=\operatorname{End}(U)$, стандартньм образом определим бимодуль ${ }_{S} U_{R}$, левый $S$-модуль $H(M)={ }_{S} \operatorname{Hom}_{R}(M, U)$ и аннуляторные отображения $\operatorname{Ann}_{H(M)}: L(M) \rightarrow L(H(M))$ и $\mathrm{Ann}_{M}: L(H(M)) \rightarrow L(M)$. Основным результатом является

ТЕОРемА. Для модуля $M_{R}$ равносильны условия:

1) $M$ является $\mathrm{AB} 5{ }^{*}$-модулем и $\mathrm{QFD}$-модулем;

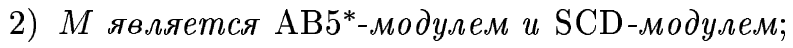

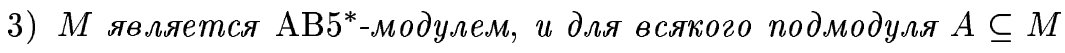
фактормодуль $A / J(A)$ конечно порохсден;

4) $M$ является $\mathrm{AB} 5{ }^{*}$-модулем, и для каждого подмодуля $A \subseteq M$ существует такой коконечный подмодуль $B \subseteq M$, ито $A \subseteq B$ $u \operatorname{Soc}(B / A)=0$;

5) модуль $H(M)$ линейно компактен, причем отображения $\operatorname{Ann}_{H(M)}: L(M) \rightarrow L(H(M)) \quad u \quad \mathrm{Ann}_{M}: L(H(M)) \rightarrow L(M)$ являются взаимно обратными решеточными антиизоморфизмами;

6) М решеточно антиизоморфен некоторому линейно компактному модулю над подходящим кольчом;

7) $М$ решеточно изоморфен некоторому линейно компактному модулю над подходящим кольцом.

Доказательству теоремы предпошлем несколько лемм, дополняя по мере надобности список обозначений и определений.

Отождествив модули $E_{i}$ с соответствуюшими подмодулями в $U_{R}$, рассмотрим подкольцо $T \subseteq S$, образованное всеми $\varphi \in S$, удовлетворяющими условию: для всякого $j \in I$ найдется такое конечное подмножество $\Xi(\varphi, j) \subseteq I$, что $\varphi\left(E_{j}\right) \subseteq \sum_{i \in \Xi(\varphi, j)} E_{i}$. Естественно возникающий бимодуль ${ }_{T} U_{R}$ позволяет ассоциировать с модулем $M_{R}$ левьй $T$-модуль ${ }_{T} \operatorname{Hom}_{R}(M, U)$ и его подмодуль $D(M)$, состоящий из всех таких $f \in \operatorname{Hom}_{R}(M, U)$, что $\operatorname{Im} f \subseteq \sum_{i \in \Theta(f)} E_{i}$ для подходящего конечного 
подмножества $\Theta(f) \subseteq I$. Аналогично случаю модуля $H(M)$ установим соответствие Галуа между $L(M)$ и $L(D(M))$ с помощью аннуляторных отображений $\operatorname{Ann}_{D(M)}: L(M) \rightarrow L(D(M))$ и $\operatorname{Ann}_{M}: L(D(M)) \rightarrow L(M)$, определяемых условиями

$$
\operatorname{Ann}_{D(M)}(A)=\{f \in D(M) \mid \operatorname{Ker} f \supseteq A\}
$$

и

$$
\operatorname{Ann}_{M}(V)=\bigcap_{f \in V} \operatorname{Ker} f
$$

для всех $A \in L(M)$ и $V \in L(D(M))$. Употребление обозначения $\mathrm{Ann}_{M}$ в двух различных ситуациях не приведет к путанице, так как ни в одном из нижеследующих утверждений конструкции $H(M)$ и $D(M)$ не будут использоваться одновременно.

ЛЕмма 1 [4]. Для модуля $M_{R}$ равносильны условия:

1) $M$ является $\mathrm{AB} 5^{*}$-модулем;

2) отображсения $\mathrm{Ann}_{D(M)}: L(M) \rightarrow L(D(M))$ и $\mathrm{Ann}_{M}: L(D(M))$ $\rightarrow L(M)$ являются взаимно обратными решеточными антиизоморфизмами;

3) М решеточно антиизоморфен некоторому модулю над подходящим кольиом.

Условимся через $L^{*}$ обозначать решетку, дуальную к решетке $L$. Класс решеток назьвается абстрактным, если он замкнут относительно изоморфных образов. Из леммы 1 непосредственно вытекает следующий принцип двойственности для АВ5*-модулей.

СлЕДСТВИЕ 1. Если $\Phi-$-абстрактный класс решеток, то равносильны условия:

1) $L(M) \in \Phi$ для любых кольца $R$ и $\mathrm{AB} 5^{*}{ }_{-м о д у л я ~} M_{R}$;

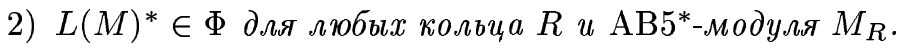

ЗАмЕчАниЕ 1. Флери [5] и Варадараян [6] предложили подходы к дуализации понятия конечномерного по Голди модуля, отличные от подхода Такеучи. Поскольку все три свойства коконечномерности выражаются в терминах решетки подмодулей модуля, для АВ 5 -модуля они оказьваются равносильньми ввиду следствия 1. В отсутствии условия АВ5* эти понятия не равносильны [7]. Подмодули AB5*-модуля наследуют условие AB5*. Поэтому при замене в определении SCD-модуля коконечномерности (в смысле Такеучи) его подмодулей на коконечномерность в смысле Флери или Варадараяна приходим к свойствам модуля, которые для AB5*-модуля равносильны условию SCD. 
Лемма 2. Для любых АВ5 ${ }^{*}$ модуля $M_{R}$ и подмодуля $A \subseteq M$ фактормодуль $A / J(A)$ вполне приводим.

ДоКАЗАТЕЛЬСТВО. Поскольку все подмодули $\mathrm{AB} 5{ }^{*}$-модуля $M_{R}$ также являются АВ5*-модулями, достаточно установить полную приводимость модуля $M / J(M)$. Обратим внимание на очевидное свойство решетки $L(M)$ : всякий подмодуль модуля $\operatorname{Soc}(M)$ выделяется прямым слагаемым в $\operatorname{Soc}(M)$. Дуализируя это условие и применяя следствие 1 , заключаем, что $M / J(M)$ вполне приводим.

Для произвольных модуля $M_{R}$ и подмодуля $V$ модуля $H(M)$ определим отображения $\beta(V): V \rightarrow H\left(M / \operatorname{Ann}_{M}(V)\right)$ и $\gamma(V): H(M) / V \rightarrow$ $H\left(\operatorname{Ann}_{M}(V)\right)$, положив $(f) \beta(V)\left(x+\operatorname{Ann}_{M}(V)\right)=f(x)$ и $(g+V) \gamma(V)(y)$ $=g(y)$, где $f \in V, x \in M, g \in H(M)$ и $y \in \operatorname{Ann}_{M}(V)$.

ЛЕмма 3. Если $M_{R}$ является $\mathrm{AB} 5{ }^{*}$-модулем $и$ QFD-модулем, то

1) отображсения $\mathrm{Ann}_{H(M)}: L(M) \rightarrow L(H(M))$ и $\mathrm{Ann}_{M}: L(H(M))$ $\rightarrow L(M)$ являются взаимно обратными решеточными антиизоморфизмами;

2) для всякого подмодуля $V \subseteq H(M)$ отображсения $\beta(V): V \rightarrow$ $H\left(M / \operatorname{Ann}_{M}(V)\right)$ u $\gamma(V): H(M) / V \rightarrow H\left(\operatorname{Ann}_{M}(V)\right)$ являются изоморфизмами $S$-модулей;

3) модуль $H(M)$ линейно компактен.

ДоКАЗАТЕЛЬСТВо. 1) В силу теоремы $2.19^{*}$ из [8] требуется лишь убедиться в $M$-конечной копорожденности наименьшего инъективного кообразующего $U_{R}$, т.е. в конечной копорожденности модуля $\operatorname{Im} f$ для любого $f \in H(M)$. Действительно, $\operatorname{Soc}(\operatorname{Im} f)$ является сушественным подмодулем в $\operatorname{Im} f$, потому что $\operatorname{Soc}\left(U_{R}\right)$ существен в $U_{R}$. Кроме того, $\operatorname{Soc}(\operatorname{Im} f)$ конечно порожден, так как $\operatorname{Im} f$ конечномерен в смысле Голди. Остается учесть предложение 21.3(1) из [2].

2) Легко проверяется с помошь 1).

3) Проведем рассуждение, предложенное для доказательства предложений $47.10(2)(\mathrm{ii})$ и $47.10(3)(\mathrm{ii})$ в [2]. Пусть $\left(V_{\lambda}\right)_{\lambda \in \Lambda}-$ направленное вниз семейство подмодулей модуля $H(M)$. Согласно 2), имеют место изоморфизмы $S$-модулей $\beta\left(V_{\lambda}\right): V_{\lambda} \rightarrow H\left(M / \operatorname{Ann}_{M}\left(V_{\lambda}\right)\right)$ и $\gamma\left(V_{\lambda}\right): H(M) / V_{\lambda} \rightarrow H\left(\operatorname{Ann}_{M}\left(V_{\lambda}\right)\right)$. Поскольку $\left(\operatorname{Ann}_{M}\left(V_{\lambda}\right)\right)_{\lambda \in \Lambda}-$ направленное вверх семейство подмодулей модуля $M$, можем написать точную последовательность

$$
0 \rightarrow \lim _{\longrightarrow} \operatorname{Ann}_{M}\left(V_{\lambda}\right) \rightarrow M \rightarrow \underset{\lim }{\longrightarrow} M / \operatorname{Ann}_{M}\left(V_{\lambda}\right) \rightarrow 0 .
$$


$\Phi$ унктор $H=\operatorname{Hom}_{R}(-, U)$ из категории правых $R$-модулей в категорию левых $S$-модулей точен. Это позволяет использовать его для построения коммутативной диаграммы

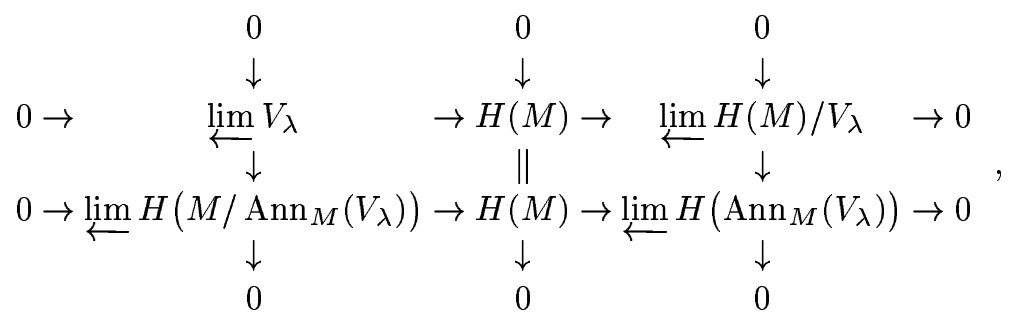

нижняя строка и столбцы которой точны. Но тогда точна и верхняя строка, и доказательство завершается применением теоремы 29.7 из [2].

ДОКАЗАТЕЛЬСТВО ТЕОРЕМЫ. 1) $\Leftrightarrow 2)$ ВЫтекает из следствия 1 и того факта, что условия QFD и $\mathrm{SCD}$, выраженные как свойства решетки $L(M)$, оказываются дуальными.

$1) \Leftrightarrow 3)$ Как известно (см. [9, лемма]), модуль $M$ является QFD-модулем тогда и только тогда, когда он удовлетворяет следующему легко переводимому на язык свойств решетки $L(M)$ условию: цоколь каждого фактормодуля модуля $M$ конечно порожден. Дуализируя это условие и привлекая следствие 1 , вьводим, что $\mathrm{AB} 5{ }^{*}$-модуль $M$ является QFD-модулем тогда и только тогда, когда для всякого подмодуля $A \subseteq M$ фактормодуль $A / J(A)$ конечно копорожден. Остается заметить, что конечная копорожденность модуля $A / J(A)$ равносильна его конечной порожденности в силу предложения 20.8 из [2] и леммы 2.

1) $\Leftrightarrow 4)$ Для того, чтобы $M$ являлся QFD-модулем, необходимо и достаточно, чтобы для каждого подмодуля $A \subseteq M$ сушествовал такой конечно порожденный подмодуль $B \subseteq A$, что $J(A / B)=A / B$ (см. [9, теорема]). Дальнейшее очевидно ввиду следствия 1 .

1) $\Rightarrow 5$ ) Содержится в лемме 3 .

5) $\Rightarrow 6$ ) Очевидно.

$6) \Rightarrow 2$ ) Если модуль $M$ решеточно антиизоморфен некоторому линейно компактному модулю $N$ над подходяшим кольцом, то, во-первых, $M$ является AB 5 -модулем по лемме 1 . Во-вторых, $M$ является SCD-модулем, так как ввиду теоремы 29.8 из [2], $N$ является QFD-модулем.

$1) \Rightarrow 7)$ Пусть, как и выше, $U_{R}$ - наименьший инъективньй кообразуюший категории правых $R$-модулей, $S=\operatorname{End}\left(U_{R}\right)$ и $H^{(R)}(M)=$ $H(M)=\operatorname{Hom}_{R}(M, U)$ - левый $S$-модуль. Пусть, далее, ${ }_{S} V-$ наименьший инъективный кообразующий категории левых $S$-модулей, 
$T=\operatorname{End}\left({ }_{S} V\right)$ и $H^{(S)}\left(H^{(R)}(M)\right)=\operatorname{Hom}_{S}\left(H^{(R)}(M), V\right)$ - правый $T$-модуль. По теореме 29.8 из [2] модуль $H^{(R)}(M)$ является АВ5*-модулем и QFD-модулем. Положим ${ }_{S} N=H^{(R)}(M)$ и $Q_{T}=H^{(S)}(N)$. Применяя лемму 3 к каждому из модулей $M$ и $N$, получаем взаимно обратные решеточные изоморфизмы $\operatorname{Ann}_{Q} \operatorname{Ann}_{N}: L(M) \rightarrow L(Q)$ и $\operatorname{Ann}_{M} \operatorname{Ann}_{N}: L(Q) \rightarrow L(M)$ (допуская вольность обозначений, в первом случае через $\mathrm{Ann}_{N}$ обозначаем аннуляторное отображение $\operatorname{Ann}_{N}: L(M) \rightarrow L(N)$, а во втором $\left.A_{N}: L(Q) \rightarrow L(N)\right)$.

$7) \Rightarrow 1)$ Ясно ввиду того, что всякий линейно компактный модуль является AB5*-модулем и QFD-модулем (см. [2, теорема 29.8]). Теорема доказана.

ЗАмЕчАниЕ 2. Как легко видеть, лемма 3 и теорема остаются справедливыми при замене в их формулировках модуля $H(M)=\operatorname{Hom}_{R}(M, U)$ на левый $C$-модуль $\operatorname{Hom}_{R}(M, X)$, где $X_{R}$ - произвольньй инъективньй кообразуюший с сушественным цоколем и $C=\operatorname{End}\left(X_{R}\right)$.

Из теоремы немедленно извлекается следующий аналог следствия 1 , представляющий из себя двойной принцип двойственности: а) для линейно компактных модулей; б) для модулей, удовлетворяющих условиям AB5* и QFD.

СлЕДСТВИЕ 2. Если $\Phi$ - абстрактный класс решеток, то равносильны условия:

1) $L(M) \in \Phi$ для любых кольца $R$ и линейно компактного модуля $M_{R}$

2) $L(M) \in \Phi$ для любых кольиа $R$ и модуля $M_{R}$, являющегося $\mathrm{AB} 5{ }^{*}$-модулем $и$ QFD-модулем;

3) $L(M)^{*} \in \Phi$ для любых кольиа $R$ и линейно компактного модуля $M_{R}$

4) $L(M)^{*} \in \Phi$ для любых кольца $R$ и модуля $M_{R}$, являющегося $\mathrm{AB} 5{ }^{*}$-модулем и QFD-модулем.

В [10] автором анонсировано

СлЕДСТВИЕ 3. ДЛя кольца $R$ равносильны условия:

1) кольцо $R$ имеет конечное число неизоморфных простых правых R-модулей

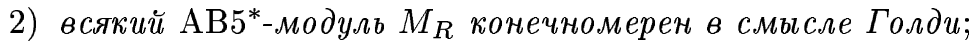

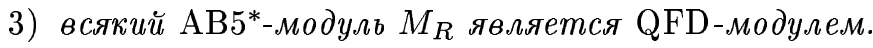


ДоКАЗАТЕЛЬСТво. $1 \Rightarrow 3$ ) В силу теоремы 47.9 из [2] и леммы 2 для всякого подмодуля $A \subseteq M$ фактормодуль $A / J(A)$ оказывается вполне приводимым модулем, все однородные компоненты которого конечно порождены. Отсюда с помощью 1) заключаем, что $A / J(A)$ конечно порожден, после чего остается применить теорему.

3) $\Rightarrow 2$ ) Очевидно.

$2) \Rightarrow 1)$ Пусть $\left(P_{i}\right)_{i \in I}$-семейство всех представителей классов изоморфных простых правых $R$-модулей и $W=\bigoplus_{i \in I} P_{i}$. Ясно, что $W$ имеет автодуальную решетку подмодулей, и следовательно, является АВ5*-модулем. Но тогда $W$ конечномерен в смысле Голди, так что множество $I$ конечно.

Модуль называется иепным, если решетка его подмодулей является цепью. Кольцо $R$ назьвается цепным справ $a$, если модуль $R_{R}$ является цепным. Аналогично определяются AB5*-кольи с справа, QFD-кольца справа и линейно компактные справа кольиа.

ЗАмЕчАниЕ 3. Поскольку правое АВ5*-кольцо полусовершенно (см. [2, теорема 47.9]), из следствия 3 вытекает следуюший известный результат (см. [11, предложение 4]): правое АВ5*-кольцо является правым QFD-кольцом. Поэтому, согласно теореме, равносильны условия: 1$) R$ является АВ5*-кольцом справа; 2) модуль $R_{R}$ решеточно изоморфен некоторому линейно компактному модулю над подходящим кольцом. Вообще говоря, АВ5*-модуль не обязан быть конечномерным в смысле Голди. Подтверждающим это примером может служить построенный в доказательстве следствия 3 модуль $W_{R}$ в случае, когда кольцо $R$ имеет бесконечное число неизоморфных простых правых $R$-модулей. Существуют и $\mathrm{QFD}$-модули, не являющиеся $\mathrm{AB} 5$-модулями, например, $\mathbb{Z}_{\mathbb{Z}}$, где $\mathbb{Z}$ - кольцо целых чисел. Наконец, чтобы ответить на вопрос о возможности определения линейно компактного модуля в терминах решетки его подмодулей, приведем пример модуля, решеточно изоморфного некоторому линейно компактному модулю над проходящим кольцом, но не являющегося линейно компактным. Так как всякий цепной модуль является $\mathrm{AB} 5{ }^{*}$-модулем и $\mathrm{QFD}$-модулем, требуемьгм примером является модуль $R_{R}$, где $R$ - цепное справа кольцо, не являющееся линейно компактньм справа. Примеры таких колец можно найти, например, в [12].

Автор благодарен А. В. Михалеву и А. А. Туганбаеву за ценные обсуждения и замечания. 


\section{СПИСОК ЦИТИРОВАННОЙ ЛИТЕРАТУРЫ}

[1] Vámos P. Classical rings // J. Algebra. 1975. V. 34. №1. P. 114-129.

[2] Wisbauer R. Foundations of Module and Ring Theory. Philadelphia: Gordon and Breach Science Publishers, 1991.

[3] Takeuchi T. On cofinite-dimensional modules // Hokkaido Math. J. 1976. V. 5. №1. P. 1-43.

[4] Бродский Г. М. О дуализмах в модулях и условии АВ5* // УМН. 1983. T. 38. № 2. C. 201-202.

[5] Fleury P. A note on dualizing Goldie dimension // Canad. Math. Bull. 1974. V. 17. №6. P. 511-517.

[6] Varadarajan K. Dual Goldie dimension // Commun. Algebra. 1979. V. 7. № 6. P. 565-610.

[7] Varadarajan K. Modules with supplements // Pacif. J. Math. 1979. V. 82. P. 559-564.

[8] Бродский Г. М. Функторы Нот и структуры подмодулей // Тр. ММО. 1983. T. 46. C. $164-186$.

[9] Camillo V. P. Modules whose quotients have finite Goldie dimension // Pacif. J. Math. 1977. V. 69. № 2. P. 337-338.

[10] Бродский Г. М. О модулях с условием AB5* // XVI Всесоюз. алгебр. конф. Тезисы. Ч. 2. Л., 1981. С. 18-19.

[11] Lemonnier B. AB5* et la dualité de Morita // C. R. Acad. Sci. Ser. A-B. 1979. V. 289. № 2. P. A47-A50.

[12] Brandal W. Commutative rings whose finitely generated modules decompose // Lect. Notes Math. 1979. V. 723. P. 1-116. 